\title{
Farmacocinética de la cefalexina administrada por vía oral conjuntamente con enalapril en caninos adultos
}

\author{
Prados, A.P.; Kreil, V.; Monfrinotti, A.; Paes, J.; Hallu, R. \\ Cátedra de Farmacología, Facultad de Ciencias Veterinarias, Universidad de Buenos Aires, \\ Av. Chorroarín 280, Buenos Aires, Argentina. Tel. 4524-8459. E-mail: aprados@fvet.uba.ar

\begin{abstract}
Resumen
Prados, A.P.; Kreil, V.; Monfrinotti, A.; Paes, J.; Hallu, R.: Farmacocinética de la cefalexina administrada por vía oral conjuntamente con enalapril en caninos adultos. Rev. Vet. 28: 2, 99-102, 2017. El objetivo de este estudio fue caracterizar la farmacocinética de la cefalexina administrada por vía oral a caninos y determinar si la administración conjunta con enalapril produce modificaciones en la misma. Se trabajó con 6 caninos mestizos sanos. Cada uno recibió una dosis única de $25 \mathrm{mg} / \mathrm{kg}$ de una suspensión de cefalexina (grupo 1) o cefalexina 20 minutos después de administrada una dosis oral única de $0,5 \mathrm{mg} / \mathrm{kg}$ de enalapril (grupo 2), con un diseño cross over (3x3). Las concentraciones plasmáticas de cefalexina se determinaron mediante el método microbiológico. El T $>$ CIM fue siempre superior al $60 \%$ del intervalo posológico de 12 horas para ambos grupos. Los parámetros farmacocinéticos se compararon mediante el test de Wilcoxon $(\mathrm{p} \leq 0,05)$. Los resultados obtenidos se expresaron como media \pm desvío estándar y para los grupos 1 y 2 fueron respectivamente: Cmax $27,66 \pm 4,20$ y $26,51 \pm 6,04 \mu \mathrm{g} / \mathrm{ml}, T \max 1,41 \pm 0,58$ y $1,58 \pm 0,58 \mathrm{~h}, \mathrm{~T}_{1 / 2} 1,55 \pm 0,12$ y $1,62 \pm 0,22 \mathrm{~h}$, $\mathrm{ABC}_{0-\infty} 110,8 \pm 10,98$ y $110,6 \pm 17,64 \mu \mathrm{g} \cdot \mathrm{h} / \mathrm{ml}$, TMR $3,19 \pm 0,39$ y $3,46 \pm 0,55 \mathrm{~h}$. No se encontraron diferencias estadísticamente significativas entre los parámetros farmacocinéticos de cefalexina obtenidos en cada grupo, por lo cual nuestros resultados sugieren que el tratamiento simultáneo con ambas drogas no modifica la farmacocinética del antibiótico.
\end{abstract}

Palabras clave: canino, cefalexina, enalapril, interacción.

\begin{abstract}
Prados, A.P.; Kreil, V.; Monfrinotti, A.; Paes, J.; Hallu, R.: Pharmacokinetics of orally administered cephalexin together with enalapril in adult dogs. Rev. Vet. 28: 2, 99-102, 2017. The purpose of the present study was to describe oral cephalexin pharmacokinetics in healthy dogs and to investigate if previous administration of enalapril affects its disposition. Six mixed breed dogs were included in this study. Each dog received oral $25 \mathrm{mg} / \mathrm{kg}$ cephalexin (group 1) or cephalexin 20 minutes after oral $0.5 \mathrm{mg} / \mathrm{kg}$ enalapril (group 2), with a 2 week wash out period, in a crossover design $(3 \times 3)$. Cephalexin plasma concentrations were determined by microbiological assay. T $>$ MIC was over $60 \%$ of dosing interval for both groups. Pharmacokinetic parameters were compared performing Wilcoxon's paired $t$ test $(\mathrm{p} \leq 0.05)$. Results are reported as mean \pm standard deviation. Group 1: Cmax 27.66 $\pm 4.20 \mu \mathrm{g} / \mathrm{ml}$, Tmax $1.41 \pm 0.58 \mathrm{~h}, \mathrm{~T}_{1 / 2} 1.55 \pm 0.12 \mathrm{~h}, \mathrm{AUC} 0-\infty 110.8 \pm 10.98 \mu \mathrm{g} \cdot \mathrm{h} / \mathrm{ml}$, and MRT $3.19 \pm 0.39 \mathrm{~h}$. Group 2:

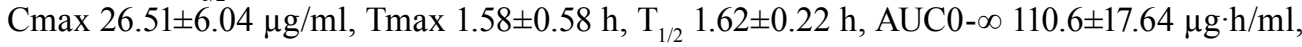
and MRT $3.46 \pm 0.55 \mathrm{~h}$. No statistical differences were found in cephalexin pharmacokinetic parameters between experiences. Consequently, enalapril, when co-administered orally with cephalexin, doesn't modify the pharmacokinetic of the antibiotic.
\end{abstract}

Key words: dog, cephalexin, enalapril, interaction.

\section{INTRODUCCIÓN}

La cefalexina es una cefalosporina de primera generación utilizada con frecuencia en medicina veterinaria, debido a su baja toxicidad y buena actividad contra bacterias gram-positivas como estreptococos y

Recibido: 14 octubre 2016 / Aceptado: 15 marzo 2017 Proyectos UBACYT 2011-2014 y 2014-2017. Presentado a XLV Reun.Soc.Arg.Farmacol.Experim., Mar del Plata 2013. estafilococos. Luego de la administración por vía oral, su absorción está mediada principalmente por el sistema de transporte de membrana PepT1, expresado en la membrana apical de las células epiteliales del duodeno, yeyuno e íleon ${ }^{2}$.

Por lo tanto, la expresión y disponibilidad del sistema PepT1 son factores importantes para la biodisponibilidad oral de este fármaco. Los antibióticos betalactámicos proporcionan una actividad bactericida dependiente del tiempo. Varios estudios han determi- 
nado que el tiempo durante el cual las concentraciones plasmáticas del fármaco permanecen por encima de las concentraciones inhibitorias mínimas (CIM) del organismo patógeno ( $>\mathrm{CIM})$ es el índice farmacocinético/ farmacodinámico (pk/pd) que mejor predice su eficacia antibacteriana ${ }^{12}$.

Por ello, cambios en las concentraciones plasmáticas pueden afectar el resultado clínico de los tratamientos con cefalexina. Los fármacos inhibidores de la enzima convertidora de angiotensina (IECA), como el enalapril, son ampliamente utilizados en caninos con patología cardiovascular. Habitualmente cuando se prescribe un IECA, es indicado de por vida, esto se debe a la notable mejoría de los signos clínicos y de la calidad de vida del paciente, como consecuencia principalmente de sus efectos vasodilatadores y de inhibición de la liberación de aldosterona.

Distintos estudios han demostrado que los IECAs, al igual que los betalactámicos, son sustrato del sistema de transporte PepT1 ${ }^{7}$ pudiendo producirse en consecuencia, una competencia por dicho mecanismo de absorción oral cuando dos drogas pertenecientes a estos grupos son administradas simultáneamente.

Debido a que no es infrecuente la presentación de algún proceso infeccioso que justifique la administración de cefalexina en un paciente cardiópata tratado con enalapril, el propósito de este estudio fue investigar si la administración previa de enalapril afecta la biodisponibilidad oral de la cefalexina en caninos y, de haber cambios, si se modifica el T>CIM.

\section{MATERIAL Y MÉTODOS}

Animales. Se utilizaron 6 caninos adultos mestizos (peso promedio de $12.9 \pm 1.38 \mathrm{~kg}$ y edad promedio 4,5 años), obtenidos de los caniles de la Facultad de Ciencias Veterinarias, Universidad de Buenos Aires. Los animales se encontraban en buen estado de salud, determinado por exámenes físicos, análisis sanguíneos y urinarios. Ninguno de los caninos tenía antecedentes de alergia a beta-lactámicos. Este trabajo fue evaluado y aprobado por el Comité Institucional de Cuidado y Uso de Animales de Laboratorio (CICUAL) de la Facultad de Ciencias Veterinarias, Universidad de Buenos Aires.

Protocolo. Los animales fueron sometidos a un ayuno sólido de 8 horas previo a la administración de las drogas. Cada canino fue pesado antes de recibir los tratamientos. Las drogas administradas fueron cefalexina $25 \mathrm{mg} / \mathrm{kg}$ en suspensión oral (Cefalexina 100 $\mathrm{mg} / \mathrm{ml}^{\mathbb{\circledR}}$ suspensión oral Argentia, Argentina) y enalapril $0,5 \mathrm{mg} / \mathrm{kg}$ (Lotrial ${ }^{\circledR}$ comprimidos, Roemmers Argentina) ambas por vía oral. Cada canino recibió cefalexina sola (grupo 1) y cefalexina 20 minutos luego de la administración de enalapril (grupo 2), con un diseño crossover. El período de lavado fue de 2 semanas entre cada grupo. Se extrajeron muestras de sangre $(1,5 \mathrm{ml})$ en tubos heparinizados, mediante un catéter colocado en la vena cefálica antebraquial derecha, a los siguientes tiempos: 0,$16 ; 0,33 ; 0,5 ; 0,75 ; 1 ; 1,5 ; 2 ; 2,5 ; 3,5 ; 5,5$; 7,5; 9,5 y 11,5 h luego de la administración de la cefalexina. Las muestras fueron inmediatamente centrifugadas y el plasma fue conservado a $-20^{\circ} \mathrm{C}$ hasta su procesamiento.

Método analítico. Las concentraciones plasmáticas de cefalexina se midieron por un método microbiológico previamente descripto ${ }^{1}$ utilizando Kocuria rhizophila ATCC 9341 como cepa patrón. La curva estándar se realizó en plasma canino $\left(\mathrm{r}^{2}=0,99\right)$. El límite de cuantificación fue $1,56 \mu \mathrm{g} / \mathrm{ml}$ y el límite de detección fue $0,79 \mu \mathrm{g} / \mathrm{ml}$. El coeficiente de variación intra/inter día y la exactitud del método (medida como desviación) fueron $<10 \%$ en el rango de concentraciones estudiadas $(50-1,56 \mu \mathrm{g} / \mathrm{ml})$.

Análisis farmacocinético. Se utilizó el programa de computación PCNONLIN 4.0 (SCI Software, Lexington, KY, USA) para calcular los parámetros farmacocinéticos de la cefalexina a partir de la curva concentración plasmática vs tiempo de cada animal, mediante un análisis no compartimental y de acuerdo a las ecuaciones clásicas ${ }^{8}$, excepto la concentración máxima (Cmax) y el tiempo máximo (Tmax), que se obtuvieron a partir de las concentraciones plasmáticas observadas. La vida media de eliminación $\left(\mathrm{T}_{1 / 2 \lambda}\right)$ se obtuvo del cociente $0,693 / \lambda$, donde $\lambda$ es la pendiente de la fase terminal de eliminación determinada por la regresión lineal de cuadrados mínimos de los últimos 4-5 puntos de la fase terminal de la curva de disposición. El área bajo la curva desde el tiempo 0 hasta la última concentración medida $\left(\mathrm{ABC}_{0-\mathrm{f}}\right)$ se calculó por la regla de los trapezoides lineales y fue extrapolada al infinito $\left(\mathrm{ABC}_{0-\infty}\right)$ de acuerdo a $\mathrm{ABC}_{0-\mathrm{t}}+\mathrm{C}_{\mathrm{t}} / \lambda$, donde $\mathrm{C}_{\mathrm{t}}$ es la última concentración medida. El tiempo medio de residencia (TMR) se calculó como $\mathrm{AUMC} / \mathrm{ABC}_{0-\infty}$, donde AUMC es el área bajo la curva del producto del tiempo y concentración plasmática de la droga versus el tiempo desde el tiempo cero a infinito. El T $>$ CIM fue determinado para cada animal mediante la observación de la curva de disposición plasmática. Se consideró 2 $\mu \mathrm{g} / \mathrm{ml}$ como punto de corte para bacterias susceptibles como los estreptococos beta-hemolíticos, Staphylococcus aureus, S. pseudointermedius y Escherichia coli ${ }^{5}$.

Análisis estadístico. Los parámetros farmacocinéticos obtenidos para cada grupo se compararon mediante el test de Wilcoxon siendo el nivel de significación $\mathrm{p}<0,05$.

\section{RESULTADOS}

Los tratamientos en ambos grupos fueron bien tolerados, no habiéndose observado reacciones adversas que pudieran relacionarse a la administración de los fármacos. En la Tabla 1 se exponen los parámetros farmacocinéticos de la cefalexina administrada vía oral 
Tabla 1. Parámetros farmacocinéticos de cefalexina (grupo 1) y cefalexina-enalapril) (grupo 2). Datos en $\overline{\mathrm{x}} \pm \mathrm{DE}$.

\begin{tabular}{lcc}
\hline parámetro & grupo 1 & grupo 2 \\
\hline Cmax $(\mu \mathrm{g} / \mathrm{ml})$ & $27,66 \pm 4,20$ & $26,51 \pm 6,04^{\text {n.s. }}$ \\
$\operatorname{Tmax}(\mathrm{h})$ & $1,41 \pm 0,58$ & $1,58 \pm 0,58^{\text {n.s. }}$ \\
$\lambda\left(\mathrm{h}^{-1}\right)$ & $0,44 \pm 0,03$ & $0,43 \pm 0,06^{\text {n.s. }}$ \\
$\mathrm{T}_{1 / 2}(\mathrm{~h})$ & $1,55 \pm 0,12$ & $1,62 \pm 0,22^{\text {n.s. }}$ \\
$\mathrm{ABC}_{0-\mathrm{t}}(\mu \mathrm{g} \cdot \mathrm{h} / \mathrm{ml})$ & $105,4 \pm 10,80$ & $104,8 \pm 16,39^{\text {n.s. }}$ \\
$\mathrm{ABC}_{0-\infty}(\mu \mathrm{g} \cdot \mathrm{h} / \mathrm{ml})$ & $110,8 \pm 10,98$ & $110,6 \pm 17,64^{\text {n.s. }}$ \\
$\operatorname{TMR}(\mathrm{h})$ & $3,19 \pm 0,39$ & $3,46 \pm 0,55^{\text {n.s. }}$ \\
\hline
\end{tabular}

Cmax, concentración máxima; Tmax, tiempo máximo; $\lambda$, constante de eliminación terminal; $T_{1 / 2}$, vida media de eliminación; $A B C_{0-t}$, área bajo la curva concentración plasmática - tiempo desde el tiempo cero hasta el último punto medido; $A B C_{0-\infty}$ área bajo la curva concentración plasmática - tiempo desde el tiempo cero hasta infinito; TMR, tiempo medio de residencia; n.s., no significativo.

a caninos, a una dosis de $25 \mathrm{mg} / \mathrm{kg}$ (grupo 1), y cefalexina luego de un pre-tratamiento con $0,5 \mathrm{mg} / \mathrm{kg}$ de enalapril (grupo 2).

Las curvas de disposición de la cefalexina construidas a partir de los valores de concentración plasmática en función del tiempo se detallan en la Figura 1. No se observaron diferencias estadísticamente significativas entre los parámetros farmacocinéticos de la cefalexina obtenidos en ambos grupos. El T $>$ CIM fue siempre superior al $60 \%$ del intervalo posológico de 12 horas para ambos grupos.

\section{DISCUSIÓN}

Dado que la administración conjunta de dos o más fármacos puede afectar la absorción, distribución y/o eliminación de alguno de ellos o de ambos, este trabajo se propuso estudiar la farmacocinética de la cefalexina administrada en combinación con enalapril. Los fármacos se administraron según las dosis recomendadas en la bibliografía y por los laboratorios productores de las mismas ${ }^{14}$.

La farmacocinética de la cefalexina administrada por distintas vías en caninos ha sido ampliamente descripta $^{3,4,6,15}$. Los parámetros farmacocinéticos obtenidos en este estudio son similares a los hallados por otros grupos de investigación cuando administraron la cefalexina a caninos por vía oral ${ }^{3,4,6}$.

Estudios previos demostraron que la administración de cefalexina con otros fármacos puede inducir cambios en su cinética. Trabajos en pacientes humanos hallaron que la co-administración por vía oral de cefalexina con ranitidina o cefalexina con omeprazol produjo un retraso en la absorción del antibiótico que se vio reflejado en una prolongación del tiempo máximo y un menor porcentaje de T>CIM ${ }^{11}$.

La cefalexina administrada por vía oral junto con metformina en seres humanos condujo a un aumento en la concentración máxima y el $\mathrm{ABC}$, así como un menor clearence de la metformina ${ }^{10}$. En otro estudio se investigó la posibilidad de una interacción farmacocinética entre el captopril y la cefalosporina cefradina, ambos administrados por vía oral a pacientes humanos, no hallando cambios significativos en la misma ${ }^{7}$.

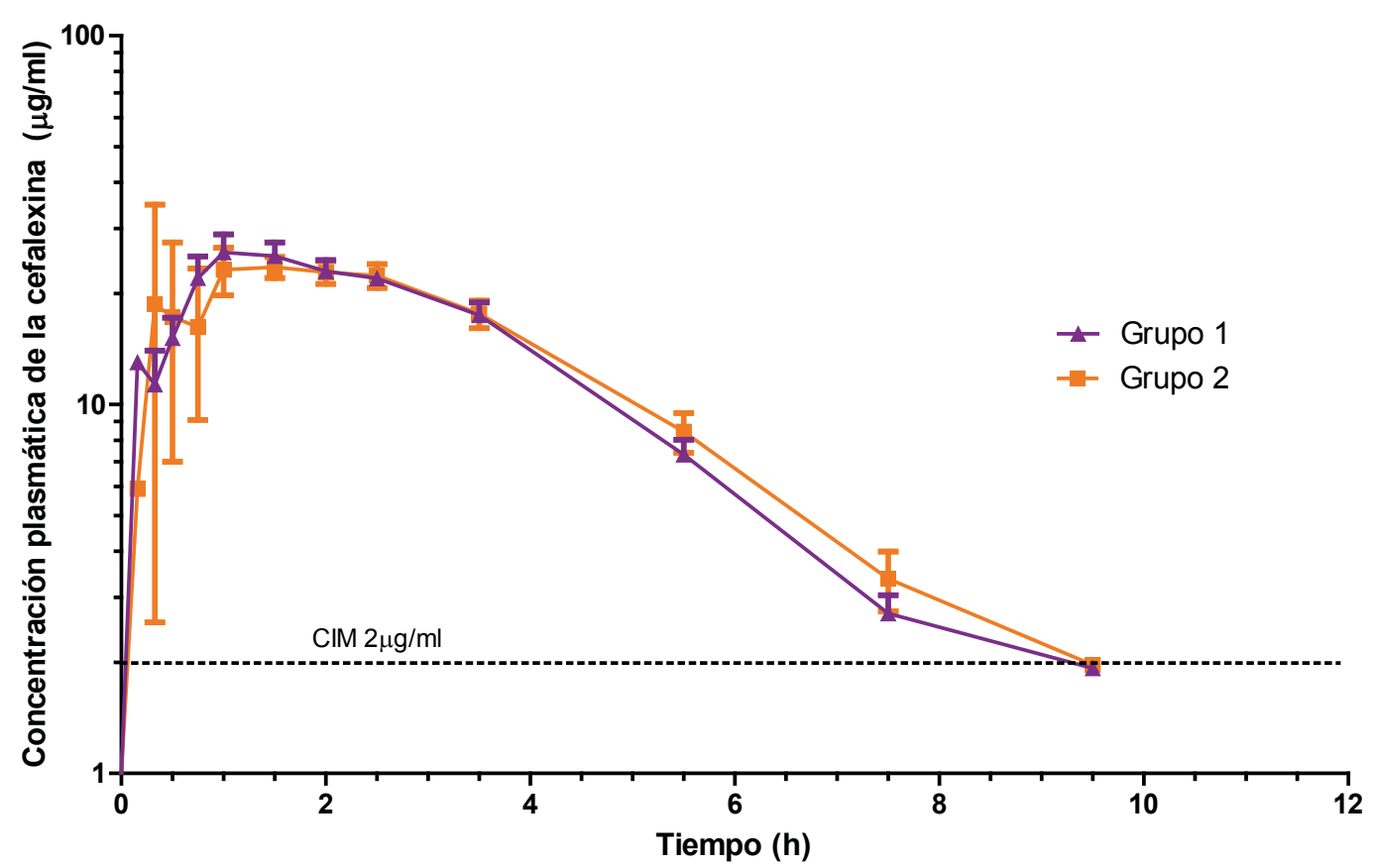

Figura 1. Concentraciones plasmáticas medias de cefalexina obtenidas luego de una dosis única oral de $25 \mathrm{mg} /$ $\mathrm{kg}$ (grupo 1) y, luego del pre-tratamiento con $0,5 \mathrm{mg} / \mathrm{kg}$ de enalapril por vía oral (grupo 2) en 6 caninos. Las barras verticales representan el DS. 
La administración simultánea de cefalexina y quinapril por vía oral a ratas produjo modificaciones en las fases de absorción y de eliminación del antibiótico, que resultaron en un aumento del $\mathrm{ABC}$ de la cefalexina ${ }^{13}$. Otra investigación en ratas observó una disminución del clearence del cefadroxilo cuando se administra conjuntamente con cefalexina, ambos por vía endovenosa ${ }^{9}$.

Administrando una dosis única por vía oral y analizando los datos mediante un modelo no compartimental, nuestros resultados demostraron que no se produjeron modificaciones en la farmacocinética de la cefalexina administrada en combinación con enalapril.

Por lo tanto, se podría concluir que no sería necesaria una modificación en la dosis del antibiótico si se emplea en forma conjunta con IECA (inhibidores de la enzima convertidora de angiotensina). Sin embargo, nuestro diseño experimental presenta una limitación importante, ya que no contempla la administración crónica de enalapril, habiéndose administrado en una única dosis por vía oral.

Agradecimientos. Este trabajo forma parte del Proyecto UBACYT 20020100100698 (2011-2014) y UBAСуT 20020130100615BA (2014-2017). Se agradece a los Veterinarios Fernando Suárez Belzoni, Nicolás Porta y Esteban Martín, así como a la Srta. Silvana Bournasell, por la ayuda brindada durante el muestro de los animales.

\section{REFERENCIAS}

1. Bennet JV, Brodie JL, Benner EJ, Kirby WM. 1966. Simplified accurate method for antibiotic assay of clinical specimens. Appl Microbiol 14: 170-177.

2. Buyse $\mathbf{M}$ et al. 2001. PepT1-mediated epithelial transport of dipeptides and cephalexin is enhanced by luminal leptin in the small intestine. J Clin Invest 108: 1483-1493.

3. Campbell BG, Rosin E. 1998. Effect of food on absorption of cefadroxil and cephalexin in dogs. J Vet Pharm \& Therap 21: 418-420.

4. Carli S, Anfossi P, Villa R, Castellani G, Mengozzi G, Montesissa C. 1999. Absorption kinetics and bioavailability of cephalexin in the dog after oral and intramuscular administration. $J$ Vet Pharm \& Therap 22: 308-313.
5. Clinical and Laboratory Standards Institute. 2013. Performance standards for antimicrobial susceptibility testing; twenty-third informational supplement. M100S23. Wayne, Pennsylvania USA: CLSI; 2013.

6. Crosse R, Burt DG. 1984. Antibiotic concentration in the serum of dogs and cats following a single oral dose of cephalexin. Vet Rec 115: 106-107.

7. Foster DR et al. 2009. Lack of interaction between the peptidomimetic substrates captopril and cephradine. $J$ Clin Pharm 49: 360-367.

8. Gibaldi M, Perrier D. 1982. Farmacocinética, Editorial Reverté S.A., Barcelona, España, p. 182-184.

9. Granero L, Gimeno MJ, Torres F, Chesa J, Peris JE. 1994. Studies on the renal excretion mechanisms of cefadroxil. Drug Metab \& Disposit 22: 447-450.

10. Jayasagar G, Krishna M, Chandrasekhar K, Madhusudan C, Madhusudan RY. 2002. Effect of cephalexin on the pharmacokinetics of metformin in healthy human volunteers. J Drug Metab \& Drug Interact 19: 41-48.

11. Madaras K, Michas P, George M, May MP, Adejare A. 2004. A randomized crossover study investigating the influence of ranitidine or omeprazole on the pharmacokinetics of cephalexin monohydrate. J Clin Pharm 44: 13911397.

12. McKellar QA, Sanchez-Bruni SF, Jones DG. 2004. Pharmacokinetic/ pharmacodynamics relationships of antimicrobial agent drugs used in veterinary medicine. $J$ Vet Pharm \& Therap 27: 503-514.

13. Padoin C, Tod M, Perret G, Petitjean O. 1998. Analysis of the pharmacokinetic interaction between cephalexin and quinapril by a nonlinear mixed-effect model. Antimicrob Agents \& Chemoth 42: 1463-1469.

14. Plumb DC. 2002. Veterinary Drug Handbook, $4^{\text {th }}$ edition, Pharma Vet Publishing, Minnesota, USA, 993 p.

15. Silley P, Rudd AP, Symington WM, Talt AJ. 1988. Pharmacokinetics of cephalexin in dogs and cats after oral, subcutaneous and intramuscular administration. Vet Rec 122: 15-17. 